\title{
Far-from-equilibrium effects of electric and electromagnetic fields in ceramics synthesis and processing
}

\author{
B. Reeja-Jayan and Jian Luo, Guest Editors
}

Electric and electromagnetic fields can promote far-from-equilibrium chemical reactions, phase transformations, and microstructural evolution. On the one hand, both direct electric fields and time-varying electromagnetic fields can change the atomic and microscale structure of materials in ways that differ from conventional methods. On the other hand, ultrafast densification in a matter of seconds (e.g., in flash sintering) and electrically induced microstructural evolution open up new opportunities for materials processing. In many cases, the external fields absorbed within a material may result in "nonthermal" effects. In other cases, thermal effects dominate, but applied fields can still influence the microstructural evolution or generate nonequilibrium defects. Consequently, new behavior evolves such as ceramics that become ductile due to high densities of dislocations created by the far-from-equilibrium processing. Many open scientific questions remain about the fundamental mechanisms underlying such interactions of external fields with matter. From an industrial standpoint, processing advanced materials using externally applied fields can have a smaller energy footprint compared to conventional methods and as such will have a profound impact on society.

\section{Introduction}

Externally applied electric and electromagnetic fields can enhance processes for materials synthesis and processing. ${ }^{1-3}$ Examples include microwave radiation-assisted crystallization and sintering ${ }^{4-9}$ as well as electric field/current-enabled synthesis and sintering. ${ }^{10-16}$ Interest in such field-assisted methods originates primarily for two main reasons. Figure 1a compares the temperature-time profiles of several techniques used for ceramic synthesis and sintering. ${ }^{1}$ All field-assisted ceramic synthesis and sintering methods occupy the low (furnace/environment) temperature and fast kinetics (short time) region shown in green, compared to conventional processes shown in the orange circles. In many instances, external fields can invoke interesting changes in the atomic and microscale structure of materials as well as phenomena, which cannot be normally achieved by conventional methods. In addition, fields can also invoke far-from-equilibrium, ultrafast processing via ultrahigh heating rates, opening up new opportunities for materials processing. These possibilities make fieldassisted methods exciting, particularly from the perspective of materials discovery and energy-efficient materials fabrication.
Field-assisted synthesis has been widely applied to engineer various ceramic compositions with precise control over size and morphology (Figure 1b) ${ }^{17,18}$ Additionally, there is a lot of interest in low-temperature processing and integration techniques for ceramics, such as cold sintering. ${ }^{19-21}$ Applying external fields such as microwave radiation ${ }^{2-24}$ provides similar opportunities to integrate ceramics that conventionally require high-processing temperatures with soft materials, such as polymers with lower melting points, enabling applications such as flexible electronics and sensors. Furthermore, modifying atomic structure can impact functional properties. For example, electric fields can trigger the emergence of metastable crystal structures ${ }^{25}$ and unexpected properties such as increased electronic conductivity ${ }^{26}$ and ductility. At room temperature, micropillars of flash-sintered $\mathrm{TiO}_{2}$ could deform plastically up to a strain of $8 \%$, unlike conventionally sintered samples that fractured elastically at $2 \%$ strain (Figure 1c) ${ }^{27}$ We do not fully understand if these abnormal albeit technologically appealing phenomena can be attributed simply to ultrahigh heating/cooling rates that emerge during synthesis or if far-from-equilibrium effects of a nonthermal 


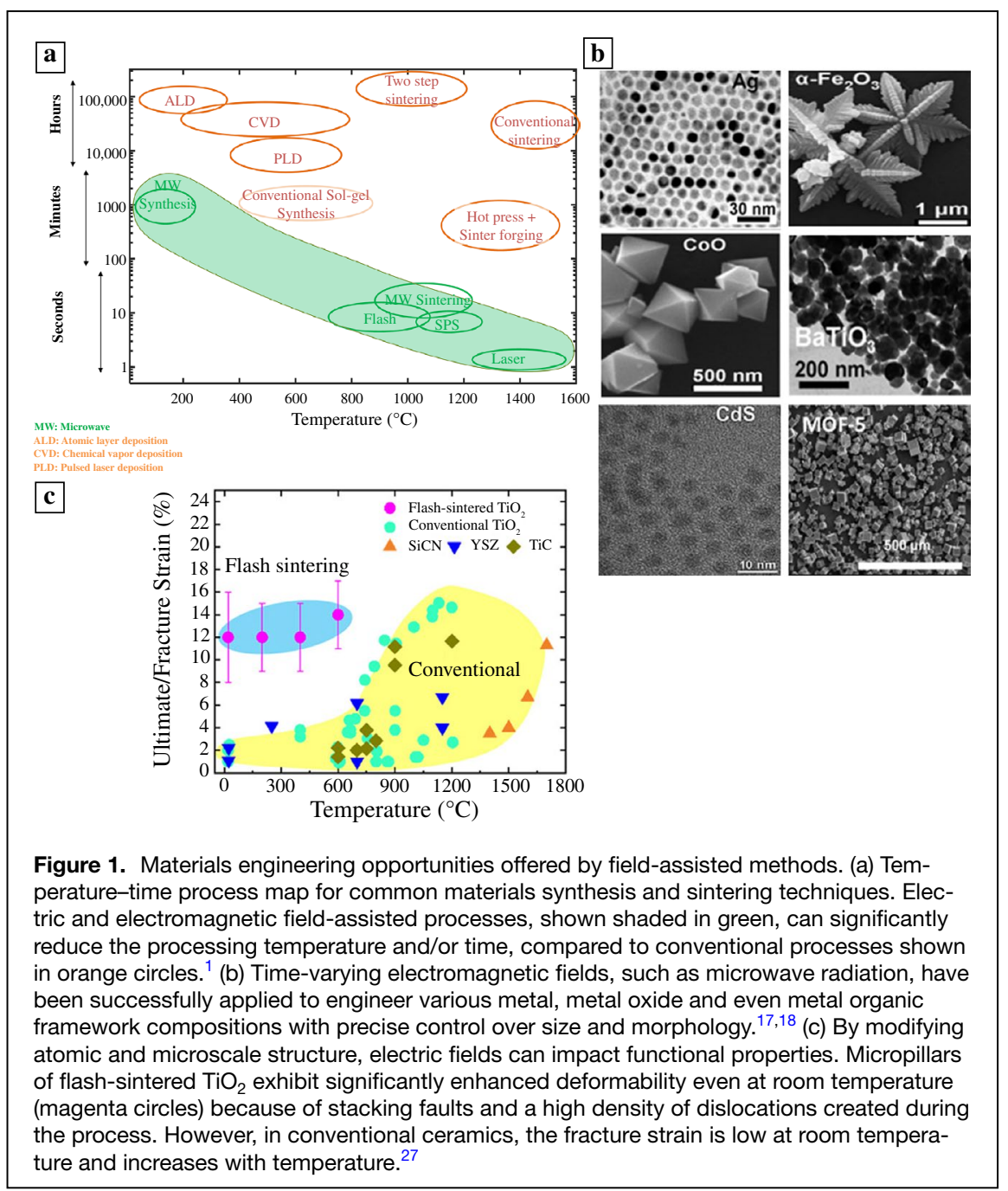

other reviews. We believe such a discussion with help enrich the breadth of this special issue.

\section{Flash and other ultrafast sintering}

In 2010, Raj and colleagues invented "flash sintering," 10 where they used an applied electric field to initiate a "flash" and subsequently rapid densification of $3 \mathrm{~mol} \% \mathrm{Y}_{2} \mathrm{O}_{3}$-stabilized $\mathrm{ZrO}_{2}$ (3YSZ) in $\sim 5 \mathrm{~s}$ at a nominal furnace temperature as low as $850^{\circ} \mathrm{C}$. Raj et al.'s pioneering work ${ }^{10}$ immediately attracted great scientific and technological interests. Researchers worldwide soon reported the successful application of flash sintering in a wide range of ceramic materials. ${ }^{11-16}$ The Raj et al. article in this issue provides both historical and recent perspectives on flash sintering and related phenomena. $^{28}$

Recently, ultrafast densification in seconds has also been achieved by rapid thermal annealing (RTA ${ }^{29}$ and ultrafast high-temperature sintering (UHS) ${ }^{30}$ both with radiative heating from surfaces to achieve high heating rates of $\sim 10^{2} \mathrm{~K} / \mathrm{s}$ akin to flash sintering (Figure $2 \mathrm{a}-\mathrm{c}$ ), but without running electric currents through the specimens.

nature, such as the hypothesized field-induced nucleation of defects, are involved. ${ }^{1-3,17}$

The articles in this issue of MRS Bulletin focus on direct electric fields, particularly for densification and sintering. Nevertheless, many of the observed effects are similar across the various field-assisted methods such as those using timevarying electromagnetic fields such as microwaves. These articles review recent successes in the use of electric fields (flash sintering) to achieve high-quality, dense ceramics at lower temperatures, as well as progress made to date on in situ diagnostics and multiscale modeling efforts. Collectively, these articles focus on advancing the understanding of structural transformations and microstructural evolution under external fields to accelerate technological development in future, energy-efficient manufacturing, and discovery of new materials.

Instead of only repeating and summarizing the in-depth discussions that are already in these articles, this article will also briefly discuss other areas and (sometimes different) scientific viewpoints that have either not or been less covered in the

\section{Fundamentals of ultrafast sintering-thermal effects}

Flash sintering ${ }^{2,10}$ as well as alterative ultrafast sintering schemes such as RTA ${ }^{29}$ or $\mathrm{UHS}^{30}$ (Figure $2 \mathrm{~b}-\mathrm{c}$ ) offer potential technological opportunities. Therefore, an in-depth understanding of underlying mechanisms is desirable. Here, the three key scientific questions are:

1. How does a "flash" start?

2. What enables rapid densifications?

3. What are the electric field/current effects on sintering and microstructural evolution?

On the one hand, it was suggested that flash sintering is associated with an avalanche generation of point (Frenkel) defects $^{28,33}$ that can produce electroluminescence ${ }^{33-35}$ and flash initiated at a critical power density. ${ }^{36}$ On the other hand, it was alternatively proposed that a flash may start as a thermal runaway in many cases ${ }^{37-39}$ and the ultrafast heating rates on 


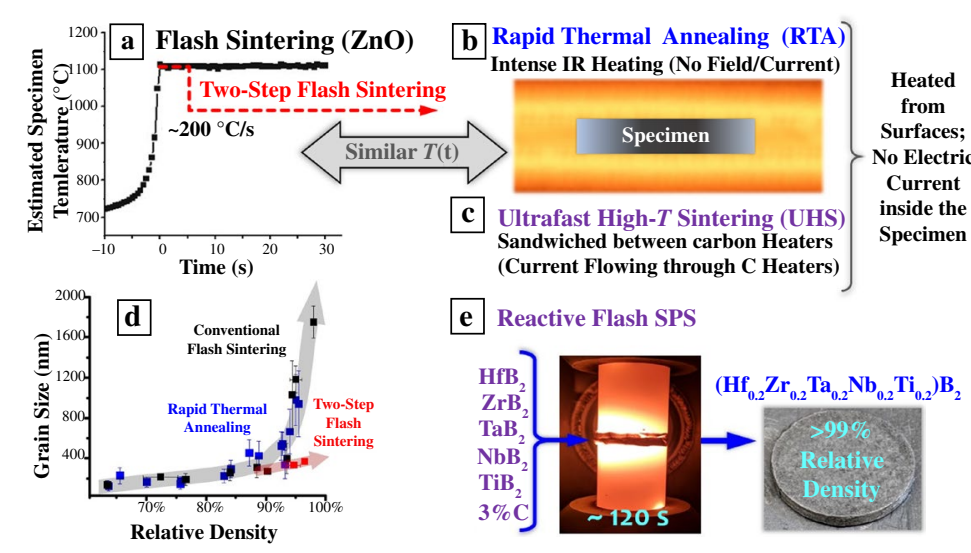

Figure 2. "Flash" sintering and other ultrafast sintering methods. (a) Estimated temperature profile, $T(t)$, for flash sintering of $\mathrm{ZnO} . .^{14,29}$ (b) Rapid thermal annealing (RTA) using intense IR heating to mimic the $T(t)$ profile of flash sintering, but without an applied electric field, can attain similar ultrafast densification rate as flash sintering. ${ }^{29}$ This critical comparison experiment suggested that the ultrahigh heating rates (of $\sim 200^{\circ} \mathrm{C}$ for both flash sintering and RTA in this case) enable ultrafast densification in $<30 \mathrm{~s}{ }^{29}$ (c) Most recently, ultrafast high-temperature sintering (UHS) was developed as a general method to sinter ceramics in seconds by heating a thin specimen sandwiched between two carbon stripes as heaters, which can achieve similar ultrahigh heating rates. ${ }^{30}$ (d) A careful comparison showed that RTA and flash sintering also exhibit similar grain size versus relative density curves. ${ }^{29}$ However, a two-step flash sintering, with a $T(t)$ profile schematically illustrated by the red dashed curved in panel (a), can densify specimens with suppressed grain growth. ${ }^{14,31}$ (e) Reactive flash spark plasma sintering (SPS) can synthesize a single-phase high-entropy metal diboride (representing perhaps one of the most difficult-to-sinter ceramics) from a mixture of raw binary boride powders and densify it to $>99 \%$ of theoretical density in 2 min. $^{32}$

It is important to identify the key factor(s) enabling the ultrafast densification. Zhang et al. reported a critical experiment in 2017 to demonstrate that comparable ultrafast densification rates can be achieved for $\mathrm{ZnO}$ specimens by (1) flash sintering (with an applied electric field) and (2) rapid thermal annealing (RTA) (using infrared heating without an applied electric field) with similar heating rates of $\sim 200^{\circ} \mathrm{C} / \mathrm{s}$ (Figure $2 \mathrm{a}-\mathrm{b}$ ). ${ }^{29}$ A careful comparison showed that RTA and flash sintering led to similar grain size versus relative density curves (Figure 2d), thereby suggesting similar underlying kinetic mechanisms. ${ }^{29}$ This study ${ }^{29}$ unequivocally demonstrated that the high heating rate $(\mathrm{d} T / \mathrm{d} t)$ on the order of $10^{2} \mathrm{~K} / \mathrm{s}$ can enable ultrafast densification in seconds without an electric field. Zhang et al. further demonstrated that densification is lower if the effective heating rate was intentionally reduced, thereby confirming that the heating rate is indeed the controlling factor. ${ }^{29}$ In the same year, Ji et al. also independently reported an "ultrafast firing" experiment using an exothermic $\mathrm{Ni}-\mathrm{Al}$ reaction and reached a similar conclusion for $3 \mathrm{YSZ}^{45}$ In this issue, Raj et al. further described a thermally activated flash-sintering experiment without an applied electric field. ${ }^{28}$

Two possible mechanisms are proposed to explain how ultrahigh heating rates enable ultrafast sintering. ${ }^{14,29,45}$ First, sintering is a competition between densification and coarsening,

the order of $10^{2} \mathrm{~K} / \mathrm{s}$ enable the ultrafast densification. ${ }^{29}$ The Raj et al. article introduces the possible generation of Frenkel defects, electroluminescence, and the critical power density, ${ }^{28}$ and the article by Guillon et al. discusses fundamentals of nonthermal effects. ${ }^{40}$ This section will discuss thermal effects that can cause ultrafast sintering.

Zhang et al. originally reported that a flash can start as a coupled thermal and electric runaway in $2015 .{ }^{37}$ In this work, they also proposed a quantitative model to use the measured temperature-dependent electrical conductivity values to predict the thermal runaway temperatures. The results agree with the observed onset flash-sintering temperatures for $\sim 20$ different cases. ${ }^{14,29,37,41,42}$ Similar thermal runaway models have also been proposed independently by Todd et al. ${ }^{38}$ at about the same time and by Dong and Chen a few months later, ${ }^{39,43}$ which were validated on additional material systems. It should be noted that these studies do not exclude the possibility that a discontinuous rise in the specimen conductivity resulting from another physical process such as a phase transformation can also trigger a flash. For example, a recent study showed that flash sintering can be activated by the formation of an ion-conducting eutectic liquid or a liquid-like grain-boundary complexion in $\mathrm{Bi}_{2} \mathrm{O}_{3}$-doped $\mathrm{ZnO}$ that caused an abrupt increase in the specimen conductivity. ${ }^{44}$ where the latter is faster at lower temperatures and reduces the sintering rate drastically. Thus, an ultrahigh heating rate $(\mathrm{d} T / \mathrm{d} t)$ can bypass the low-temperature coarsening to keep the sintering driving force high. Second, it is hypothesized that ultrahigh heating rates may also lead to the formation of nonequilibrium grain-boundary structures with enhanced mass-transport rates to increase sintering rates.

These findings suggest the possibility of decoupling the electric response of specific materials to develop general ultrafast sintering technologies. In fact, the basic feasibility was already demonstrated by the RTA experiment reported in $2017,,^{29}$ albeit the maximum temperature was limited to $\sim 1100^{\circ} \mathrm{C}$ for typical RTA equipment. In a recent 2020 report, ${ }^{30}$ ultrafast high-temperature sintering (UHS) was developed as a general method to synthesize and sinter ceramics in seconds by heating a thin specimen sandwiched between two carbon stripes as the heater (Figure 2c). In UHS, the specimen is also heated by radiation from the surface, while the electric current runs through the carbon stripes around the specimen. The ultrafast densification in seconds was enabled by the high heating rate $(\mathrm{d} T / \mathrm{d} t)$ on the order of $10^{2} \mathrm{~K} / \mathrm{s}$ in all three cases: flash sintering (Figure 2a), ${ }^{10,14}$ RTA (Figure 2b), ${ }^{29}$ and UHS (Figure 2c). ${ }^{30}$ 
Several new or refined ceramic sintering technologies were also proposed and tested. First, several settings to realize flash sintering in spark plasma sintering (SPS) machines, or "flash SPS (FSPS)", were developed. ${ }^{32,46,47}$ For example, Gild et al. showed that reactive flash SPS (ReaFSPS) can synthesize a single-phase high-entropy metal diboride, which represents perhaps one of the most difficult-to-sinter ceramics, from a mixture of raw binary boride powders and densify it to $>99 \%$ relative density in $\sim 2$ min (Figure $2 \mathrm{e}$ ). ${ }^{32}$ Second, Nie et al. showed the possibility of activating a flash at room temperature with water vapor in "water-assisted flash sintering (WAFS)" to subsequently densify a $\mathrm{ZnO}$ green specimen to $\sim 98 \%$ density in $30 \mathrm{~s}^{48}$ Third, following Chen and Wang's pioneering work on two-step sintering, ${ }^{49}$ a two-step flash-sintering (TSFS) technology was developed to densify ceramics with suppressed grain growth using direct electronic controls (Figure 2d). ${ }^{31}$ Using $\mathrm{ZnO}$ as a benchmark, this TSFS was shown to be $>100 \times$ faster than the conventional two-step sintering, yet it achieved comparable results. ${ }^{31}$ Fourth, Kermani et al. demonstrated "flash cold sintering" of $\mathrm{ZnO} .{ }^{50}$ Beyond these examples, many new technologies (e.g., continuous flash sintering ${ }^{51}$ ) also exist and need to be further explored. See a recent review by Biesuz et al. ${ }^{52}$ for further discussion.

Finally, it is important to note that the previously discussed findings about thermal runaway and the role of ultrahigh heating rates in enabling ultrafast densification do not exclude the possible electric field effects. In fact, there is ample evidence that applied electric fields/currents can significantly influence both sintering and microstructural evolution, which will be discussed later.

\section{Fundamental questions-atomistic structural changes under electric and electromagnetic fields} The observations of unexplained structural effects under external fields, particularly effects not readily explained by conventional heating, open up discussion about potentially field-driven, nonthermal mechanisms. ${ }^{1-4,9,10,16,53,54}$ Indeed, changes in materials under field excitation have been observed at a range of length scales from atomistic to microstructure. An example for the former is field-driven decrystallization of materials. ${ }^{23,24,55,56}$ Bottom panel in Figure 3a shows an x-ray diffraction pattern, where the sharp peaks indicate crystalline anatase $\mathrm{TiO}_{2} \cdot{ }^{56}$ When exposed to time-varying microwave radiation at $2.45 \mathrm{GHz}$, peak intensity decreases drastically, indicating decrystallization. Interestingly, this material is becoming more amorphous even though we are heating it, instead of becoming more crystalline or undergoing a phase transformation. ${ }^{56}$ This decrystallization phenomena has been observed across different materials, including semiconductors such as $\mathrm{Si}^{55}$ and ferrimagnetic $\mathrm{Fe}_{3} \mathrm{O}_{4},{ }^{23}$ where we see loss of crystalline phase peaks after exposure to electric and magnetic fields, resulting in completely decrystallized materials. Another example comes from electric field-assisted flash sintering. ${ }^{57}$ Figure $3 \mathrm{~b}$ plots atomic displacement parameters as a function of time during sintering of $\mathrm{TiO}_{2}$. Atomic displacement parameters were obtained from synchrotron x-ray total scattering experiments and quantifies the average displacement of an atom from its expected lattice position, where higher values indicate more displacement and vice versa. As the field is cycled $\mathrm{ON}$ and OFF, the displacement of Ti atoms rises and falls along a fairly regular pattern and matches well to values, which correspond to the rise in temperature of the sample. However, the $\mathrm{O}$ displacement values rise and fall in a much less uniform pattern and reach significantly higher values. ${ }^{57}$ These as yet unexplained structural observations such as large displacement on the $\mathrm{O}$ lattice lead to hypothesis suggesting that applied fields may be promoting the formation of defects and altering mass transport. ${ }^{28,33,58,59}$

An additional structural effect associated with field-assisted methods is anisotropic lattice expansion, which is inconsistent with conventional heating. ${ }^{56}$ Figure $3 \mathrm{c}$ (top panel) shows how the lattice parameters of a crystalline $\mathrm{TiO}_{2}$ sample expand linearly with rise in temperature, as expected for isotropic expansion of the lattice under conventional heating. However, this linear trend essentially falls apart on heating using $2.45 \mathrm{GHz}$ microwave radiation, which results in a highly nonlinear, nonmonotonic expansion-contraction in the lattice parameters (bottom panel of Figure 3c). ${ }^{56}$ Note that the horizontal axis here uses time instead of temperature to account for this nonlinear heating profile. Indeed, we observe that the lattice initially shrinks followed by expansion, which could be attributed to the combined result of thermal expansion caused by temperature rise and defect-driven nonthermal contraction of the $\mathrm{TiO}_{2}$ lattice under the applied field. The resultant lattice parameter is determined by which of these factors is dominant at any given point in time. Once the field is switched off at $150 \mathrm{~min}$, both lattice parameters drop immediately, but do not return to their room temperature values, suggesting the presence of kinetically constrained defects that remain in the structure. ${ }^{56}$ Interestingly, such anisotropic lattice expansion-contraction phenomena have also been observed during flash sintering of $3 \mathrm{~mol} \%$ yttria-stabilized $\mathrm{ZrO}_{2}$ and $\mathrm{CeO}_{2}$ under a direct electric (DC) field. ${ }^{58,60}$ Figure $3 \mathrm{~d}$ shows how the lattice parameters initially expand corresponding to a rise in temperature; later diverge and become anisotropic, and remain so even after the electric field is turned off. What is common to all these examples is the idea that externally applied fields may promote structural effects at the atomistic scale. As described in the next section, external fields may also alter processing and microstructural evolution, including densification and grain growth.

\section{Fundamental questions-electric field effects on sintering and microstructure}

Applied fields can also influence morphological evolution (i.e., sintering and grain growth). The article by Guillon et al. in this issue has already discussed grain growth under electric fields. ${ }^{40}$ Here, we briefly highlight related observations. 


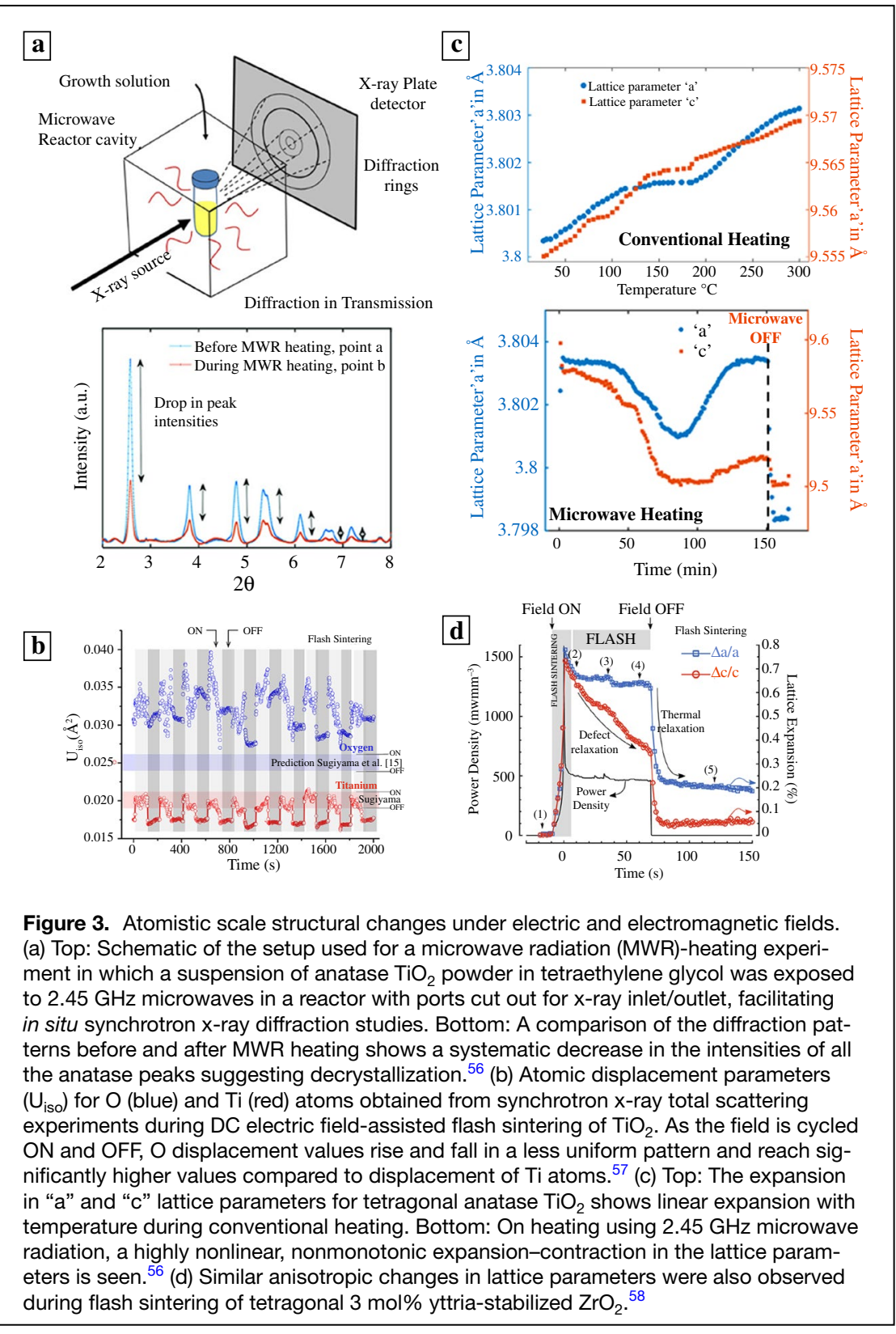

While ultrahigh heating rates can enable ultrafast sintering with and without an applied electric field, there is also evidence that large applied electric currents can promote sintering at low temperatures at least in some materials systems. Notably, Chen and co-workers unequivocally demonstrated that ionomigration of pores could lead to "electro-sintering" of $8 \mathrm{YSZ}$ at hundreds of degrees below the normal sintering temperatures. ${ }^{61-63}$ This unambiguously demonstrated a case of a significant electric current effect on enhancing the densification rates (at a high current density). It remains unknown how generalizable this mechanism is to other materials.

The effects of electric fields and currents on grain growth and microstructural developments are more intriguing. Studies by Conrad et al. showed that a relatively weak applied DC or AC field could inhibit grain growth of $3 \mathrm{YSZ}$, which they attributed to the interaction of the applied electric fields with space charges. ${ }^{64-68} \mathrm{In}$ contrast, Chen and colleagues observed enhanced grain growth on the cathode in $8 \mathrm{YSZ}, 3 \mathrm{YSZ}$, and other fluorite-type oxides, which was attributed to the electrochemically driven reduction that lowers the GB migration barriers. ${ }^{69-73}$ They further showed that an "oxygen potential transition" in the bulk can induce an abrupt (phase-like) transition in grain growth. ${ }^{73}$ Electric field effects on microstructural evolution in $\mathrm{SrTiO}_{3}$ have also been reviewed by Guillon et al. in this issue, ${ }^{40}$ so not repeated here. However, the atomic level mechanisms of how applied electric fields and currents can alter grain growth is still not well understood in general.

\section{Beyond direct electric fields}

The articles in this theme issue focus on the flash-sintering process, which typically applies direct electric fields to sinter and density ceramic specimens. However, nonequilibrium processes that occur under externally applied fields are far more diverse and can also incorporate time-varying electromagnetic (EM) fields, such as but not limited to microwave radiation. ${ }^{4-9}$ In this section, we highlight recent developments in uncovering fundamental mechanisms by which low energy microwave fields can be coupled into materials.

\section{Experimental evidence for the influence of microwave radiation on atomic structure}

Microwave ovens operating at $2.45 \mathrm{GHz}$ have been a mainstay of home and industry for more than 70 years. In the materials sciences, microwaves were shown to significantly reduce the temperatures required to assemble atoms into crystalline nanostructures. However, there remained questions about the mechanisms by which these low energy, centimeter-wavelength radiation, engaged at the atomic scale and delivered sufficient energy to drive structural changes. ${ }^{22,74,75}$ When a material absorbs microwaves energy, its polar molecules can directly couple with the oscillating electric field through dielectric polarization. ${ }^{76}$ These rotating dipoles collide with each other and result in dielectric heating. Free electrons and ions can also move 
in response to the electric field resulting in ohmic heating, which can be several times stronger in intensity than dielectric effects. ${ }^{76}$ It is, however, unclear if these interactions alone can explain how microwaves catalyze chemical reactions at low temperatures ${ }^{17,74,77}$ and drive structural changes in a variety of materials. ${ }^{4,22,56,75}$ In an example for the latter, discussed next, microwave fields induce disorder in local atomic arrangements that subsequently leads to crystallization of ceramic oxides such as $\mathrm{TiO}_{2}$ at temperatures as low as $150-160^{\circ} \mathrm{C}$. $^{75,77}$

In their experiments, Nakamura et al. used $2.45 \mathrm{GHz}$ microwave radiation to synthesize and crystallize $\mathrm{TiO}_{2}$ thin films. ${ }^{75,77}$ Microwaves selectively interact with thin $(100-150 \mathrm{~nm})$ conducting layers of indium tin oxide (ITO) on a glass substrate, allowing $\mathrm{TiO}_{2}$ films to heterogeneously nucleate, grow, and crystallize onto the substrate from a (liquid) precursor. Specifically, they show that localizing the field absorption using these conducting layers stabilizes the anatase $\mathrm{TiO}_{2}$ phase at low temperatures $\left(150-160^{\circ} \mathrm{C}\right)$. It is plausible that there is an enhancement in local field strength, which distorts local atomic structure and provides additional nucleation sites for anatase phase formation. ${ }^{4}$ Local field enhancements could also be influenced by free charge carriers present in the conducting layer coupling with the microwaves, as well as polarizability of molecules in present the precursor. ${ }^{76}$ Indeed, in situ measurements demonstrated that high $\mathrm{O}$ displacements and changes in the precursor structure can help drive the phase formation process during microwave exposure. ${ }^{4}$ Conventional heating is unable to replicate these structural effects even when utilizing similar heating rates and bulk solution temperatures. ${ }^{4,22,56}$ This suggests that changes in local electric fields and local atomic structure, which do not occur conventionally can be responsible for the effects observed during field-assisted processes.

\section{Exploring potential mechanisms}

Several additional mechanisms have also been suggested to explain the influence of microwave fields on chemical reactions, phase transformations, and microstructural evolution. Examples include field-induced ponderomotive driving forces, ${ }^{3,5,9,78,79}$ localized heating around grain boundaries, ${ }^{2,16,54}$ and the effect of ultrahigh heating rates that can radically alter mass-transport phenomena. ${ }^{29,45}$ However, an important question remains unanswered: Can microwave radiation provide sufficient energy to break chemical bonds? Microwaves at $2.4-2.5 \mathrm{GHz}$ have been shown to induce chemical and structural changes in both organic and inorganic materials. $^{4,17,22,55,56,74,75,77-79}$ However, the energy of these waves is not, in theory, sufficient to provide the $1-10 \mathrm{eV} /$ atom required to bring changes in molecular, atomic and electronic structures of metallic, semiconducting, and molecular materials. Unlike microwaves, the strong electric fields in an ultrafast laser pulses can drive electrons and the lattice away from equilibrium, resulting in lattice disorder. ${ }^{80-82}$ Once the electrons equilibrate, the unconstrained ions that are left behind remain trapped in an interstitial site, potentially generating Frenkel pair defects. Recently, femtosecond-resolution laser pulses ${ }^{83}$ were even used for crystal lattice engineering in chalcogenide glasses. A strong preferential orientation dependence on electric-field polarization was observed. ${ }^{84}$

One possibility is that free charge carriers in materials can move with the periodic cycles of microwaves, thereby amplifying the local field intensities, particularly at surfaces (e.g., particles $)^{56}$ and interfaces (e.g., thin-film growth experiments by Nakamura et al. ${ }^{75,77}$ Resistive switching experiments, in which the resistance of a dielectric material is modified by the application of an electric field, ${ }^{85}$ have indeed demonstrated that oxygen defect movement can be driven by electric fields. ${ }^{86-88}$ Another example is electrolyte gating experiments, where a voltage is applied across an oxide surface and an ionic liquid. ${ }^{89}$ Here too, local electric field intensities on the order of $10^{6}-10^{8} \mathrm{~V} / \mathrm{m}$ are seen to generate phase transitions mediated by oxygen vacancy formation. ${ }^{90-92}$ It is possible that if local electric fields on the same order of magnitude can form under microwave exposure, then they can provide a source of ionic movement or defect formation, resulting in electricfield-assisted decrystallization ${ }^{23,24,55,56}$ and even changes in mechanical behavior. ${ }^{93}$ In recent work, defect-level microstructural reconfiguration in a $\mathrm{Ti}-\mathrm{Al}$ alloy under pulsed current generated electroplastic deformation; a result that cannot be explained by just Joule heating. ${ }^{93}$

Atomistic simulations have also shown that soft, low-lying phonon modes close to the Brillouin zone edge can generate Frenkel defects in metal and oxides. ${ }^{94}$ It remains a possibility that such modes can be triggered by microwaves, leading to the observed structural changes. However, these energytransfer mechanisms remain unclear. Going forward, efforts are needed to identify the fundamental mechanisms that are common across field-assisted processing techniques spanning a range of energy/frequency levels. The insights obtained can represent an important step toward utilizing EM fields in materials design and engineering at the atomic scale. In situ characterization coupled with computational modeling efforts are critical to realize such a vision.

\section{In situ characterization}

All field-assisted processes (including synthesis and sintering) are inherently time dependent, which makes it challenging to understand underlying dynamic effects without some form of in situ monitoring while the field is being applied. ${ }^{95-98}$ Such in situ experiments become extremely important to conduct local structural characterization that can provide evidence for the formation and impacts of multiscale defects (from atomic to microscale) during these processes. ${ }^{99,100}$ Many challenges remain with regards to developing instrumentation for in situ data collection that is also compatible with field exposure. Additionally, a majority of studies relied on traditional x-ray diffraction to characterize atomic structure, which is inadequate to quantify disorder, particularly in the local atomic structure. 
Substitutional and interstitial defects, lattice distortions, dislocations, precipitates, grain boundaries, and higher order defects created during field exposure disturb the strict periodic order of an ideal crystal. Scattering contributions arising from such disorder appear in the diffuse scattering and not in sharp Bragg peaks, which arise from periodicity. Relying solely on conventional $\mathrm{x}$-ray diffraction and Bragg peaks means that we will miss these structural differences as they cannot provide us a quantitative analysis of the diffuse scattering from partially disordered and nanoscale materials. For disordered materials, total scattering methods such as real-space high-resolution pair distribution function (PDF) analysis is more relevant, because it enables structural characterization of both crystalline and noncrystalline materials by collecting and modeling data from both Bragg and diffuse scattering. ${ }^{101}$ Field-grown samples are never perfect crystals, but instead mixtures that contain varying degrees of crystallinity and disorder. ${ }^{75}$ The PDF G(r) gives the scaled probability of finding pairs of atoms separated by distance $r$, so that peaks in the PDF correspond to interatomic distances. This allows us to examine the structure of field-grown samples without assuming periodicity, enabling accurate characterization of internal and surface atomic packing, disorder, and lattice strain.

Building on their ex situ experiments on $\mathrm{TiO}_{2},{ }^{75,77}$ Nakamura et al. designed a custom-designed microwave reactor for in situ synchrotron X-ray PDF analysis of changes in atomic structure during EM field-assisted synthesis of $\mathrm{SnO}_{2}$ nanoparticles (Figure 4a). ${ }^{4}$ They found that the enhanced growth of crystalline rutile $\mathrm{SnO}_{2}$ nanoparticles during EM field exposure is predated by structural reordering of the $\mathrm{O}$ sublattice. In contrast, nanoparticles synthesized under conventional hydrothermal conditions exhibit no such structural changes and experience limited particle growth compared to EM field-assisted conditions. This change is seen clearly by monitoring the atomic displacements during synthesis (Figure 4b). ${ }^{4}$ For Sn, the displacement values start off high during the transient early stage of growth, but quickly converge to similar values as the rutile phase forms. However, for the $\mathrm{O}$ displacement, the behavior of samples with microwave exposure compared to those without are quite different. They observed a large sharp peak in the $\mathrm{O}$ displacement in samples under microwave exposure as well as the presence of a second smaller peak, whereas the conventional sample displays a single broad, less intense increase. Beyond synthesis, several other experiments that apply electric or EM fields to ceramic materials have found similar effects. During electric field-assisted sintering (flash) experiments, the atomic displacement of $\mathrm{O}$ atoms was larger than what was observed under conventional heating (Figure $3 \mathrm{~b}) .{ }^{57}$ Electric fields created metastable cubic structures in yttria-stabilized $\mathrm{ZrO}_{2},{ }^{25}$ phase transitions to oxygen deficient Magnéli phases in $\mathrm{TiO}_{2},{ }^{102}$ and enhanced mechanical properties of ceramics through defect-mediated inelastic deformation mechanisms. ${ }^{27}$ The article by Phuah et al. in this issue describes in situ studies on the temperature-dependent transition of deformation mechanisms that can produce room temperature plasticity in $\mathrm{TiO}_{2}$ from stacking faults, potentially generated under the electric field. ${ }^{103}$ 
Along with $\mathrm{O}$ displacement, changes in the surrounding (liquid) precursor can drive phase formation. This is seen when comparing the first PDF scan (start of synthesis) with the final PDF (end of synthesis) in Figure 4c. For the conventional sample, there is a slight shift, but no decrease in the $\mathrm{Sn}-\mathrm{Cl}$ peak intensity. This indicates that the $\mathrm{Sn}-\mathrm{Cl}$ bonds in the precursor $\left[\mathrm{SnCl}_{3}\left(\mathrm{H}_{2} \mathrm{O}\right)_{3}\right]^{+}$were not involved in the conventional phase formation process, and that the conventional rutile phase formation was likely driven by reorientation of existing Sn-O octahedra in the precursor. ${ }^{4}$ This result aligns with prior conventional hydrothermal synthesis studies using a similar precursor solution. ${ }^{104}$ However, under field exposure, there is a clear decrease in the $\mathrm{Sn}-\mathrm{Cl}$ peak intensity. This difference indicates a loss of highly polar $\mathrm{Sn}-\mathrm{Cl}$ bonds under field exposure and confirms that the resultant phase formation process differs from conventional synthesis and might even depend strongly on polarization effects of individual ionic species present and the atomic structure. Prior work by Youssef et al. indeed suggest that polarization effects can lower defect formation energies in metal oxides. ${ }^{105}$

\section{Modeling}

There are a number of reports on modeling and simulation of electric field effects at atomic, microstructural, and macroscopic levels during flash sintering. At the atomistic level, the possible generation of Frenkel defects (vacancy-interstitial pairs) in flash sintering remains a critical question. On the one hand, Schie et al. predicted that the field needed to generate Frenkel pairs in $\mathrm{HfO}_{2}$ (as an example) is on the order of $10^{9} \mathrm{~V} / \mathrm{m},{ }^{106}$ compared to the typical electric fields of $<10 \mathrm{~V} / \mathrm{m}$ used in flash sintering in most cases). On the other hand, Raj et al. argues that a narrow band of power density for the occurrence of flash sintering in most systems suggested a role for lattice vibrations in the flash process. ${ }^{28}$ See Vikrant et al. ${ }^{107}$ in this issue for further discussion on related phenomena.

Substantial progress has been made at the mesoscale level by García and co-workers via continuum models for both thermodynamics and kinetics. ${ }^{108-111}$ A thorough review of the modeling of flash sintering of ionic ceramics, including an outlook for future directions, is provided by Vikrant et al. in this issue. ${ }^{107}$

\section{Conclusions and future opportunities}

While we continue to make progress in our understanding of electric and EM field-driven mechanisms in materials, an important challenge remains to accurately measure local temperatures at the sites where field couples with the material. Commercially available temperature probes such as infrared sensors, thermocouples, and fiber optic probes cannot be readily deployed to measure temperature with sufficient temporal and spatial resolution, while simultaneously monitoring changes in structure and functional behavior. Some probes such as thermocouples also contain metallic components and can be ohmically heated under EM fields. Performing these measurements will require the development of new noninvasive temperature measurement techniques.

Subjecting materials to external fields, as well as the ultrafast processing itself, can trigger conditions away from equilibrium. Modeling multiscale phenomena such as defect generation will require efforts to integrate computational and data analysis techniques across multiple scales, from atomistic to microscale, as well as machine learning models. Applying the right model can be valuable for guided optimization of synthesis parameters, ${ }^{112,113}$ exploration of new regions on a process map, ${ }^{114}$ and identification of mechanisms underlying phase formation. ${ }^{115}$ The ability to collect large and timeresolved data sets during in situ monitoring of field-assisted processes creates the opportunity to incorporate such models, which can further guide us in understanding field-assisted and ultrafast processes.

The high melting temperatures of most ceramics make it impossible to print fully dense parts without high-temperature post-processing (sintering). This added step increases complexity of current additive manufacturing methods for ceramics (e.g., stereolithography) and associated thermal stresses create part shrinkage and cracking. ${ }^{116-118}$ High post-processing temperatures further make it difficult to integrate delicate materials such as polymers to realize new polymer-ceramic and metal-ceramic composites. Additive manufacturing of ceramics can become more realistic and convenient if we can print and densify complex shapes and parts. This further makes the field-assisted processes appealing because the post-processing steps currently used to densify printed ceramic parts (e.g., sintering in a furnace at high temperature for long durations) present a major bottleneck to realizing such a vision.

The article by Jones et al. in this issue identifies approaches that can reduce thermal and microstructural gradients that challenge the adoption of such emerging fieldassisted sintering techniques for manufacturing ceramics at an industrial scale. ${ }^{119}$ Machine learning-guided optimization of part shapes, "flash" electrode placement locations, and densification behavior of flash-sintered parts can also be of value here. Given an input three-dimensional model, such algorithms could learn from experimental and computational data to predict the configurations and regions on its boundary where electrodes may be placed (to apply electric field) for creating the highest densification within the object while minimizing computational cost. Integration of advanced hardware, sensors, robotic controls, and closed-loop data analysis will round out the innovations, converging fieldassisted processes toward a dynamic, intelligent, and selflearning cyber manufacturing platform.

More opportunities can present themselves as we expand our studies to material systems beyond ceramics. For instance, microwave-assisted methods are being explored for recycling plastic wastes, ${ }^{120}$ converting natural gas to value-added chemicals, ${ }^{121}$ and ambient pressure ammonia synthesis. ${ }^{122}$ Electric field-driven electrostatic interactions 
have been responsible for catalysis ${ }^{123-125}$ and for dramatically accelerating the rate of chemical reactions inside water microdroplets compared with the rates of the same reactions in bulk water. ${ }^{126}$ The formation of an electrochemical double layer that amplified local electric fields likely plays a role in the latter case and similar effects may also occur at solid-liquid interfaces during microwave-assisted synthesis. ${ }^{75}$ Developing the physical understanding and the tools for modulating the electric field locally in a reacting system in a reproducible and of course scalable manner will perhaps one day lead to precision synthesis of materials and products. This is already being called the holy grail of chemistry ${ }^{127}$ and perhaps can one day be used to engineer new states of matter for applications ranging from electronics and clean energy to materials for quantum computing and communications.

\section{Acknowledgments}

B.R.J. acknowledges support from the US Air Force Office of Scientific Research (AFOSR) Award No. FA9550-17-10120 and the US Army Research Office (ARO) Award No. W911NF1710589. J.L. gratefully acknowledges support from the Aerospace Materials for Extreme Environments program of the US Air Force Office of Scientific Research (AFOSR) under Grant No. FA9550-19-1-0327.

\section{References}

1. S.K. Jha, X.L. Phuah, J. Luo, C.P. Grigoropoulos, H. Wang, E. García, B. ReejaJayan, J. Am. Ceram. Soc. 102, 5 (2019)

2. R. Raj, M. Cologna, J.S. Francis, J. Am. Ceram. Soc. 94, 1941 (2011)

3. K.I. Rybakov, E.A. Olevsky, E.V. Krikun, J. Am. Ceram. Soc. 96, 1003 (2013)

4. N. Nakamura, L. Su, J. Bai, S. Ghose, B. Reeja-Jayan, J. Mater. Chem. A 8, 15909 (2020)

5. J.H. Booske, R.F. Cooper, S.A. Freeman, K.I. Rybakov, V.E. Semenov, Phys. Plasmas 5, 1664 (1998)

6. M. Willert-Porada, "Microwave Effects on Spinodal Decomposition," MRS Online Proceedings Library Archive 430, 403 (1996)

7. G.B. Dudley, R. Richert, A.E. Stiegman, Chem. Sci. 6, 2144 (2015)

8. G.A. Tompsett, W.C. Conner, K.S. Yngvesson, ChemPhysChem 7, 296 (2006)

9. A.G. Whittaker, Chem. Mater. 17, 3426 (2005)

10. M. Cologna, B. Rashkova, R. Raj, J. Am. Ceram. Soc. 93, 3556 (2010)

11. M. Biesuz, V.M. Sglavo, J. Eur. Ceram. Soc. 39, 115 (2019)

12. 0. Guillon, C. Elsässer, 0. Gutfleisch, J. Janek, S. Korte-Kerzel, D. Raabe, C.A. Volkert, Mater. Today 21, 527 (2018)

13. C.E.J. Dancer, Mater. Res. Express 3, 102001 (2016)

14. J. Luo, Scr. Mater. 146, 260 (2018)

15. M. Yu, S. Grasso, R. McKinnon, T. Saunders, M.J. Reece, Adv. Appl. Ceram. 116, 24 (2017)

16. J. Narayan, Scr. Mater. 69, 107 (2013)

17. M. Baghbanzadeh, L. Carbone, P.D. Cozzoli, C.0. Kappe, Angew. Chem. Int. Ed. 50, 11312 (2011)

18. J.S. Choi, W.J. Son, J. Kim, W.S. Ahn, Microporous Mesoporous Mater. 116, 727 (2008)

19. J. Guo, H. Guo, A.L. Baker, M.T. Lanagan, E.R. Kupp, G.L. Messing, C.A. Randall, Angew. Chem. Int. Ed. 55, 11457 (2016)

20. J. Guo, R. Floyd, S. Lowum, J.-P. Maria, T. Herisson de Beauvoir, J.-H. Seo, C.A. Randall, Annu. Rev. Mater. Res. 49, 275 (2019)

21. S. Grasso, M. Biesuz, L. Zoli, G. Taveri, A.I. Duff, D. Ke, A. Jiang, M.J. Reece, Adv. Appl. Ceram. 119, 115 (2020)

22. B. Reeja-Jayan, K.L. Harrison, K. Yang, C.-L. Wang, A.E. Yilmaz, A. Manthiram, Sci. Rep. 2, 1003 (2012)

23. R. Roy, R. Peelamedu, L. Hurtt, J. Cheng, D. Agrawal, Mater. Res. Innov. 6, 128 (2002)

24. R. Roy, Y. Fang, J. Cheng, D.K. Agrawal, J. Am. Ceram. Soc. 88, 1640 (2005)
25. J.M. Lebrun, G.M. Timothy, S.C.F. John, C.S. Kevin, M.K. Waltraud, R. Raj, J. Am. Ceram. Soc. 98, 1493 (2015)

26. S.K. Jha, R. Raj, J. Am. Ceram. Soc. 97, 527 (2014)

27. J. Li, J. Cho, J. Ding, H. Charalambous, S. Xue, H. Wang, X.L. Phuah, J. Jian, X. Wang, C. Ophus, T. Tsakalakos, Sci. Adv. 5, p.eaaw5519 (2019)

28. R. Raj, A. Kulkarni, J.-M. Lebrun, S. Jha, MRS Bull. 46 (1), 36 (2021)

29. Y. Zhang, J. Nie, J.M. Chan, J. Luo, Acta Mater. 125, 465 (2017)

30. C. Wang, W. Ping, Q. Bai, H. Cui, R. Hensleigh, R. Wang, A.H. Brozena, Z. Xu, J. Dai, Y. Pei, C. Zheng, G. Pastel, J. Gao, X. Wang, H. Wang, J.-C. Zhao, B. Yang, X. Zheng, J. Luo, Y. Mo, B. Dunn, L. Hu, Science 368, 521 (2020)

31. J. Nie, Y. Zhang, J.M. Chan, S. Jiang, R. Huang, J. Luo, Scr. Mater. 141, 6 (2017)

32. J. Gild, K. Kaufmann, K. Vecchio, J. Luo, Scr. Mater. 170, 106 (2019)

33. S.K. Jha, K. Terauds, J.M. Lebrun, R. Raj, J. Ceram. Soc. Jpn. 124, 283 (2016)

34. K. Naik, S.K. Jha, R. Raj, Scr. Mater. 118, 1 (2016)

35. K. Terauds, J.-M. Lebrun, H.-H. Lee, T.-Y. Jeon, S.-H. Lee, J.H. Je, R. Raj, J. Eur. Ceram. Soc. 35, 3195 (2015)

36. R. Raj, J. Am. Ceram. Soc. 99, 3226 (2016)

37. Y. Zhang, J.-I. Jung, J. Luo, Acta Mater. 94, 87 (2015)

38. R.I. Todd, E. Zapata-Solvas, R.S. Bonilla, T. Sneddon, P.R. Wilshaw, J. Eur Ceram. Soc. 35, 1865 (2015)

39. Y. Dong, I.W. Chen, J. Am. Ceram. Soc. 98, 2333 (2015)

40. O. Guillon, R.A. De Souza, T.P. Mishra, W. Rheinheimer, MRS Bull. 46 (1), 52 (2021)

41. Y. Zhang, J. Luo, Scr. Mater. 106, 26 (2015)

42. Y. Zhang, J. Nie, J. Luo, J. Ceram. Soc. Jpn. 124, 296 (2016)

43. Y. Dong, I.W. Chen, J. Am. Ceram. Soc. 98, 3624 (2015)

44. Y. Zhang, J. Nie, J. Luo, Acta Mater. 181, 544 (2019)

45. W. Ji, B. Parker, S. Falco, J.Y. Zhang, Z.Y. Fu, R.I. Todd, J. Eur. Ceram. Soc. 37, 2547 (2017)

46. E.A. Olevsky, S.M. Rolfing, A.L. Maximenko, Sci. Rep. 6, 33408 (2016)

47. S. Grasso, T. Saunders, H. Porwal, B. Milsom, A. Tudball, M. Reece, J. Am. Chem. Soc. 99, 1534 (2016)

48. J. Nie, Y. Zhang, J.M. Chan, R. Huang, J. Luo, Scr. Mater. 142, 79 (2018)

49. I.-W. Chen, X.-H. Wang, Nature 404, 168 (2000)

50. M. Kermani, M. Biesuz, J. Dong, H. Deng, M. Bortolotti, A. Chiappini, M.J Reece, V.M. Sglavo, C. Hu, S. Grasso, J. Eur. Ceram. Soc. 40, 6266 (2020)

51. E. Sortino, J.M. Lebrun, A. Sansone, R. Raj, J. Am. Ceram. Soc. 101, 1432 (2018)

52. M. Biesuz, S. Grasso, V.M. Sglavo, Curr. Opin. Solid State Mater. Sci. 24, 100868 (2020)

53. K.S. Naik, V.M. Sglavo, R. Raj, J. Eur. Ceram. Soc. 34, 4063 (2014)

54. R. Chaim, Materials 10,179 (2017)

55. A. Nozariasbmarz, K. Dsouza, D. Vashaee, Appl. Phys. Lett. 112, 093103 (2018)

56. S.K. Jha, N. Nakamura, S. Zhang, L. Su, P.M. Smith, X.L. Phuah, H. Wang, H. Wang, J.S. Okasinski, A.J.H. McGaughey, B. Reeja-Jayan, Adv. Eng. Mater 21, 1900762 (2019)

57. B. Yoon, D. Yadav, R. Raj, E.P. Sortino, S. Ghose, P. Sarin, D. Shoemaker, J. Am. Ceram. Soc. 101, 1811 (2018)

58. J.M. Lebrun, C.S. Hellberg, S.K. Jha, W.M. Kriven, A. Steveson, K.C. Seymour, N. Bernstein, S.C. Erwin, R. Raj, J. Am. Ceram. Soc. 100, 4965 (2017)

59. D. Yadav, R. Raj, J. Am. Ceram. Soc. 100, 5374 (2017)

60. S.K. Jha, H. Charalambous, H. Wang, X.L. Phuah, C. Mead, J. Okasinski, H. Wang, T. Tsakalakos, Ceram. Int. 44, 15362 (2018)

61. S.-W. Kim, S.-J.L. Kang, I.W. Chen, J. Am. Ceram. Soc. 96, 1090 (2013)

62. S.-W. Kim, S.-J.L. Kang, I.W. Chen, J. Am. Ceram. Soc. 96, 1398 (2013)

63. I.W. Chen, S.-W. Kim, J. Li, S.-J.L. Kang, F. Huang, Adv. Energy Mater. 2, 1383 (2012)

64. H. Conrad, D. Yang, Mater. Sci. Eng. A 528, 8523 (2011)

65. D. Yang, H. Conrad, Mater. Sci. Eng. A 528, 1221 (2011)

66. J. Obare, W.D. Griffin, H. Conrad, J. Mater. Sci. 47, 5141 (2012)

67. H. Conrad, J. Am. Ceram. Soc. 94, 3641 (2011)

68. D. Yang, H. Conrad, Scr. Mater. 63, 328 (2010)

69. S.W. Kim, S.G. Kim, J.I. Jung, S.J.L. Kang, I.W. Chen, J. Am. Ceram. Soc. 94, $4231(2011)$

70. Y.H. Dong, L. Qi, J. Li, I.W. Chen, Acta Mater. 126, 438 (2017)

71. Y. Dong, H. Wang, I.W. Chen, J. Am. Ceram. Soc. 100, 876 (2017)

72. Y. Dong, L. Qi, J. Li, I.-W. Chen, Acta Mater. 127, 73 (2017)

73. Y. Dong, I.W. Chen, Acta Mater. 156, 399 (2018)

74. W.C. Conner, G.A. Tompsett, J. Phys. Chem. B 112, 2110 (2008)

75. N. Nakamura, M. Terban, S.J.L. Billinge, B. Reeja-Jayan, J. Mater. Chem. A 5, 18434 (2017)

76. Principles of Electronic Materials and Devices, Third Edition, S.0. Kasap, (McGraw-Hill, 2005) 
77. N. Nakamura, J. Seepaul, J.B. Kadane, B. Reeja-Jayan, Appl. Stoch. Models Bus. Ind. 33, 314 (2017)

78. A. Nozariasbmarz, M. Hosseini, D. Vashaee, Acta Mater. 179, 85 (2019).

79. A. Malhotra, M. Hosseini, S.H. Zaferani, M. Hall, D. Vashaee, ACS Appl. Mater Interfaces 12, 50941 (2020)

80. S. Sundaram, E. Mazur, Nat. Mater. 1, 217 (2002)

81. D. Cahill, S. Yalisove, MRS Bull. 31, 594 (2006)

82. S.M. Yalisove, K. Sugioka, C.P. Grigoropoulos, MRS Bull. 41, 955 (2016)

83. D. Savytskii, B. Knorr, V. Dierolf, H. Jain, Sci. Rep. 6, 23324 (2016)

84. D. Savytskii, H. Jain, N. Tamura, V. Dierolf, Sci. Rep. 6, 36449 (2016)

85. A. Sawa, Mater. Today 11, 28 (2008)

86. D.S. Jeong, H. Schroeder, R. Waser, Phys. Rev. B 79, 195317 (2009)

87. J.J. Yang, M.D. Pickett, X. Li, D.A.A. Ohlberg, D.R. Stewart, R.S. Williams, Nat. Nanotechnol. 3, 429 (2008)

88. D.M. Evans, T.S. Holstad, A.B. Mosberg, D.R. Småbråten, P.E. Vullum, A.L. DadIani, K. Shapovalov, Z. Yan, E. Bourret, D. Gao, J. Akola, J. Torgersen, A.T.J. van Helvoort, S.M. Selbach, D. Meier, Nat. Mater. 19,1195 (2020)

89. C. Leighton, Nat. Mater. 18, 13 (2019)

90. J. Jeong, N. Aetukuri, T. Graf, T.D. Schladt, M.G. Samant, S.S.P. Parkin, Science 339, 1402 (2013)

91. N. Lu, P. Zhang, Q. Zhang, R. Qiao, Q. He, H.-B. Li, Y. Wang, J. Guo, D. Zhang, Z. Duan, Z. Li, M. Wang, S. Yang, M. Yan, E. Arenholz, S. Zhou, W. Yang, L. Gu, C.-W. Nan, J. Wu, Y. Tokura, P. Yu, Nature 546, 124 (2017)

92. S. Chen, Z. Wang, H. Ren, Y. Chen, W. Yan, C. Wang, B. Li, J. Jiang, C. Zou, Sci. Adv. 5, eaav6815 (2019)

93. S. Zhao, R. Zhang, Y. Chong, X. Li, A. Abu-Odeh, E. Rothchild, D.C. Chrzan, M. Asta, J.W. Morris, A.M. Minor, Nat. Mater. (2020), https://www.nature.com/artic les/s41563-020-00817-z.

94. M. Jongmanns, D.E. Wolf, J. Am. Ceram. Soc. 103, 589 (2020)

95. N. Nakamura, B. Reeja-Jayan, J. Mater. Res. 34, 194 (2019)

96. K.M. Ø. Jensen, H.L. Andersen, C. Tyrsted, E.D. Bøjesen, A.-C. Dippel, N. Lock, S.J.L. Billinge, B.B. Iversen, M. Christensen, ACS Nano 8, 10704 (2014)

97. D. Saha, K.M.Ø. Jensen, C. Tyrsted, E.D. Bøjesen, A.H. Mamakhel, A.-C. Dippel, M. Christensen, B.B. Iversen, Angew. Chem. Int. Ed. 53, 3667 (2014)

98. C. Tyrsted, N. Lock, K.M. Ø. Jensen, M. Christensen, E.D. Bøjesen, H. Emerich, G. Vaughan, S.J.L. Billinge, B.B. Iversen, IUCrJ 1, 165 (2014)

99. S. Tominaka, H. Yamada, S. Hiroi, S.I. Kawaguchi, K. Ohara, ACS Omega 3, 8874 (2018)

100. H. Yamada, S. Tominaka, K. Ohara, Z. Liu, T. Okubo, T. Wakihara, J. Phys. Chem. C123, 28419 (2019)

101. T. Egami, S.J.L. Billinge, Underneath the Bragg Peaks: Structural Analysis of Complex Materials (Elsevier, 2012)

102. R.J. Kamaladasa, A.A. Sharma, Y.T. Lai, W. Chen, P.A. Salvador, J.A. Bain, M. Skowronski, Y.N. Picard, Microsc. Microanal. 21, 140 (2015)

103. X.L. Phuah, J. Cho, T. Tsakalakos, A.K. Mukherjee, H. Wang, X. Zhang, MRS Bull. 46 (1), $44(2021)$

104. K.M. Ø. Jensen, M. Christensen, P. Juhas, C. Tyrsted, E.D. Bøjesen, N. Lock, S.J.L. Billinge, B.B. Iversen, J. Am. Chem. Soc. 134, 6785 (2012)

105. M. Youssef, K.J. Van Vliet, B. Yildiz, Phys. Rev. Lett. 119, 126002 (2017)

106. M. Schie, S. Menzel, J. Robertson, R. Waser, R.A. De Souza, Phys. Rev. Mater. 2, 035002 (2018)

107. K.S.N. Vikrant, X.L. Phuah, J. Lund, H. Wang, C.S. Hellberg, N. Bernstein, W. Rheinheimer, C.M. Bishop, H. Wang, R.E. Garcia, MRS Bull. 46 (1), 67 (2021)

108. K. Vikrant, W.C. Chueh, R.E. García, Energy Environ. Sci. 11, 1993 (2018)

109. K. Vikrant, H. Wang, A. Jana, H. Wang, R.E. García, NPJ Comput. Mater. 6, 1 (2020)

110. K. Vikrant, R.E. García, NPJ Comput. Mater. 5, 1 (2019)

111. K.S.N. Vikrant, W. Rheinheimer, H. Sternlicht, M. Bäurer, R.E. García, Acta Mater. 200, 727 (2020)

112. E. Kim, K. Huang, A. Tomala, S. Matthews, E. Strubell, A. Saunders, A. McCallum, E. Olivetti, Sci. Data 4, 170127 (2017)

113. P. Raccuglia, K.C. Elbert, P.D.F. Adler, C. Falk, M.B. Wenny, A. Mollo, M. Zeller S.A. Friedler, J. Schrier, A.J. Norquist, Nature 533, 73 (2016)

114. J.M. Granda, L. Donina, V. Dragone, D.-L. Long, L. Cronin, Nature 559, 377 (2018)

115. E. Kim, K. Huang, S. Jegelka, E. Olivetti, NPJ Comput. Mater. 3, 53 (2017)
116. Z.C. Eckel, C. Zhou, J.H. Martin, A.J. Jacobsen, W.B. Carter, T.A. Schaedler, Science 351, 58 (2016)

117. J.A. Gonzalez, J. Mireles, Y. Lin, R.B. Wicker, Ceram. Int. 42, 10559 (2016)

118. J. Deckers, J., Vleugels, J.P. Kruth, J. Ceram. Sci. Technol. 5, 245 (2014)

119. G.M. Jones, M. Biesuz, W. Ji, S. Fisher John, C. Grimley, C. Manière, C.E.J. Dancer, MRS Bull. 46 (1), 59 (2021)

120. X. Jie, W. Li, D. Slocombe, Y. Gao, I. Banerjee, S. Gonzalez-Cortes, B. Yao, H. AlMegren, S. Alshihri, J. Dilworth, J. Thomas, T. Xiao, P. Edwards, Nat. Catal. 3, $902(2020)$

121. X. Bai, B. Robinson, D. Shekhawat, V. Abdelsayed, J. Hu, "Microwaves in Nonoxidative Conversion of Natural Gas to Value-Added Products," in Direct Natural Gas Conversion to Value-Added Chemicals (CRC Press, Boca Raton, FL, 2020), pp. 25-52

122. C. Wildfire, V. Abdelsayed, D. Shekhawat, M.J. Spencer, Catal. Commun. 115, 64 (2018)

123. A. Warshel, Acc. Chem. Res. 14, 284 (1981)

124. M. Shetty, M.A. Ardagh, Y. Pang, O.A. Abdelrahman, P.J. Dauenhauer, ACS Catal. 13, $15(2020)$

125. C.D. Armstrong, A.R. Teixeira, React. Chem. Eng. 5, 2185 (2020)

126. X. Yan, R.M. Bain, R.G. Cooks, Angew. Chem. Int. Ed. 55, 12960 (2016)

127. R.N. Zare (Royal Society of Chemistry, 2020), https://www.chemistryworld. com/holy-grails/views/a-new-grail.

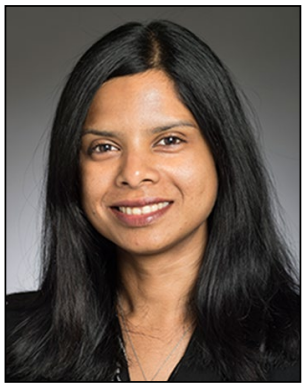

B. Reeja-Jayan is an associate professor in mechanical engineering at Carnegie Mellon University. She also holds courtesy appointments in materials science and engineering, chemical engineering, and electrical and computer engineering. Her research explores ways by which electromagnetic fields can synthesize materials hitherto unavailable to conventional synthesis routes. These low-temperature processed materials directly grow on flexible, lightweight substrates, enabling structurally integrated energy and sensing. She is a recipient of the 2018 National Science Foundation CAREER Award, 2017 Army Research Office Young Investigator Award, 2016 Air Force Office of Scientific Research Young Investigator Award, the George Tallman Ladd Research Award, and Pittsburgh Magazine's 40 Under 40 Award. Reeja-Jayan can be reached by email at bjayan@andrew.cmu.edu.

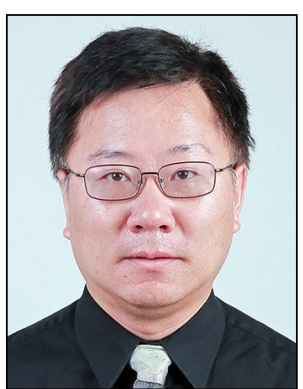

Jian Luo has been a professor of nanoengineering and materials science and engineering at the University of California, San Diego, since 2013. He received dual bachelor's degrees (one in materials science and engineering, another in electronics and computer technology) from Tsinghua University, China. He received his MS degree in materials science and engineering, and $\mathrm{PhD}$ degree in ceramics from the Massachusetts Institute of Technology. He worked in the industry for Lucent Technologies and OFS on optical fiber R\&D from 2001 to 2003. He joined the faculty of Clemson University in 2003. His current research focuses on interfaces in metals and ceramics, high-entropy and compositionally complex ceramics, novel ceramic processing, high-temperature nanoalloys, and materials for advanced energy applications. Luo's awards include a National Science Foundation CAREER Award in 2005, AFOSR Young Investigator Award in 2007, Vannevar Bush Faculty Fellow in 2014, Fellow of the American Ceramic Society in 2016, and TMS Brimacombe Medalist in 2019. Luo can be reached by email at jluo@ucsd.edu. 